\title{
New Settings for Immune Checkpoint Inhibitors in Urothelial Cancer
}

\author{
Presented by Arlene O. Siefker-Radtke, MD
}

\begin{abstract}
The advent of immune checkpoint inhibitors (ICls) has changed the game in cancer immunotherapy, specifically in the treatment of urothelial bladder cancer. Several clinical trials combining chemotherapy with ICls have resulted in approvals from the FDA and subsequent revisions within the NCCN Guidelines. The current NCCN Guidelines for Bladder Cancer reflect the most up-to-date, evidence-based data relating to the evaluation and management of urothelial bladder cancer. ICls have been incorporated into the guidelines as maintenance therapy in response to chemotherapy, sequencing after disease progression from frontline chemotherapy, and for the treatment of non-muscle-invasive bladder cancer.
\end{abstract}

J Natl Compr Canc Netw 2021;19(5.5):629-632 doi: $10.6004 /$ jnccn.2021.5007

There have been many randomized trials trying to improve on outcomes with the M-VAC (methotrexate/vinblastine/doxorubicin/cisplatin) regimen in the treatment of urothelial bladder cancer; however, according to Arlene O. Siefker-Radtke, MD, Professor of Genitourinary Medical Oncology, The University of Texas MD Anderson Cancer Center, at the NCCN 2021 Virtual Annual Conference, these attempts have been met with defeat. Specifically, Dr. Siefker-Radtke and colleagues conducted a phase III trial of M-VAC versus FU + interferon alfa-2b and cisplatin. ${ }^{1}$ The novel biochemotherapy regimen seemed to be active; however, results demonstrated that it was too toxic for general use. To date, dose-dense M-VAC and gemcitabine + cisplatin remain the typical frontline standards.

"We were so desperate to incorporate the immune system because we believed that it could help control this tumor," she said. "It really took the advent of a new class of drugs - immune checkpoint inhibitors [ICIs] - for us to finally see these new approvals and advances in the treatment of patients with urothelial cancer."

\section{Reasons to Combine Chemotherapy With Immunotherapy}

According to Dr. Siefker-Radtke, the early focus was to compare chemotherapy with immunotherapy for the treatment of metastatic disease. The open-label, international, phase III KEYNOTE-045 trial evaluated the safety and efficacy of second-line pembrolizumab in patients with platinum-refractory advanced urothelial carcinoma. ${ }^{2}$ The results, published in 2017, were instrumental in defining the role of immunotherapy in this setting. "We see potential evidence suggesting there is a group that does better initially with chemotherapy, but it is the durability of immunotherapy in the long term that makes immunotherapy quite attractive," she stated. "This may impact how we incorporate the ICIs with systemic chemotherapy."

Dr. Siefker-Radtke also noted that chemotherapy may impact antigen presentation and enhance immune responses. In fact, based on the results of a 2019 study, patients with high levels of CD19-positive antigen-presenting cells seemed to derive an overall survival (OS) benefit from adjuvant cisplatin-based chemotherapy. ${ }^{3}$ These data suggest the immune system may be involved in the setting of chemotherapy for urothelial tumors.

Additionally, another study published in 2019 revealed that PD-L1 expression levels appeared to increase in patients with non-small cell lung cancer who underwent neoadjuvant chemotherapy. ${ }^{4}$ The ability to target PD-L1 may overcome a mechanism of resistance associated with poor outcomes, according to Dr. Siefker-Radtke.

\section{Reasons Not to Combine Chemotherapy With Immunotherapy}

Chemotherapeutic agents have been found to possess immunosuppressive properties as well, according to Dr. Siefker-Radtke. She also stated that neutropenia and lymphopenia are frequently reported with agents such as gemcitabine, which could impact the ability to induce an immune response. A phase II study showed evidence of immune-related toxicities after treatment with the CTLA4 antibody ipilimumab in combination with gemcitabine + cisplatin. $^{5}$ This chemoimmunotherapy regimen did not appear to impact OS.

Furthermore, another study suggested that chemotherapy may promote tolerance to the immune response. 


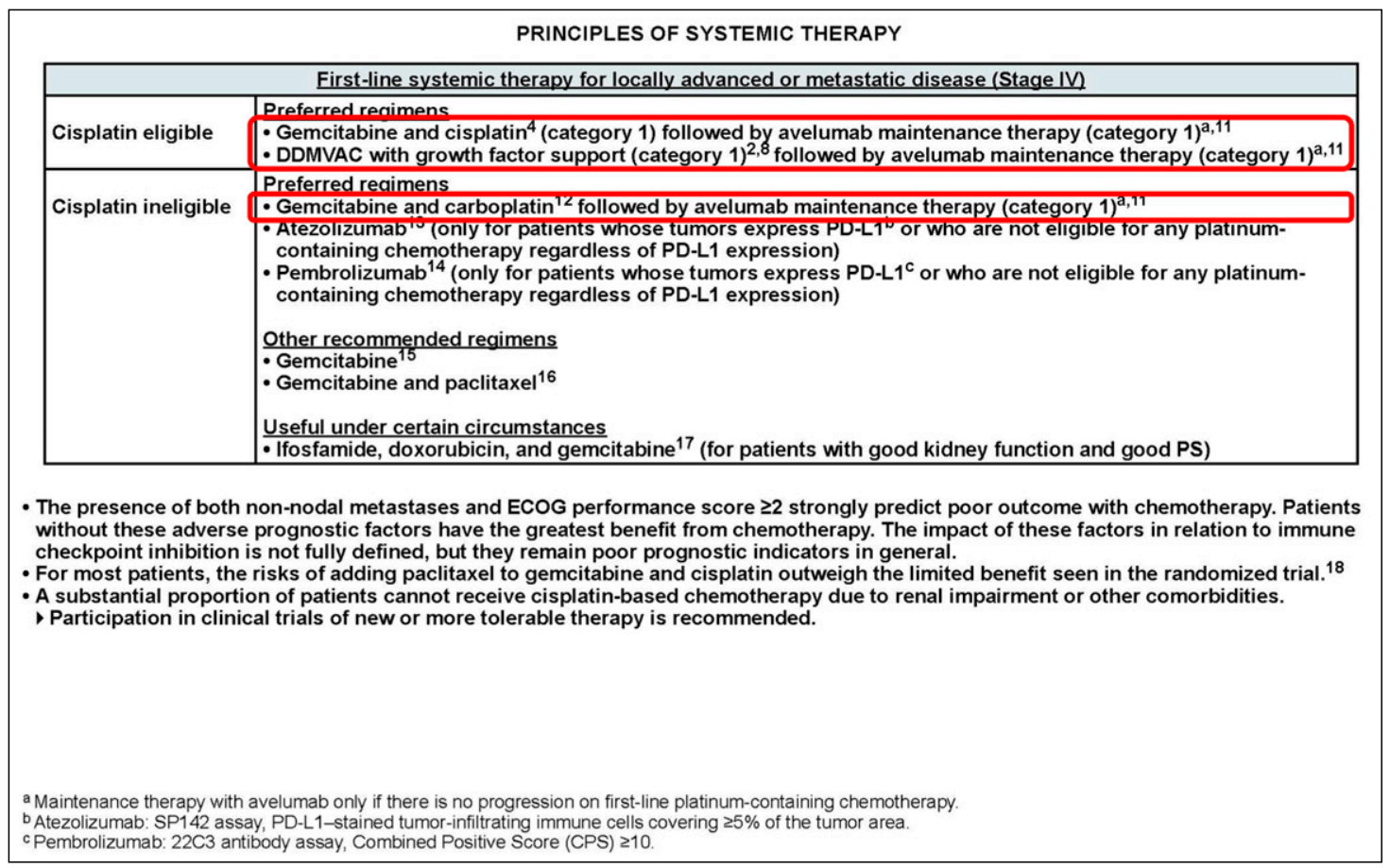

Figure 1. First-line systemic therapy for locally advanced or metastatic disease (stage IV) (BL-G 2 of 7). From the NCCN Guidelines for Bladder Cancer. Version 1.2021. To view the most recent version, visit NCCN.org.

(0)2021 National Comprehensive Cancer Network (NCCN), All rights reserved. This illustration may not be reproduced in any form without the express written permission of NCCN.

In the presence of cisplatin, there seemed to be a reduction in the maturation of dendritic cells, and in the dendritic cell stimulation of CD4-negative and CD8-positive $\mathrm{T}$ cells. There also appeared to be an increase in the number of regulatory cells that may inhibit or block the immune response. ${ }^{6}$

\section{Methods of Combination: Combination Strategy}

ICIs have been administered concurrently with chemotherapeutic agents in the setting of urothelial cancer. Early results from a trial combining neoadjuvant gemcitabine + cisplatin with pembrolizumab were presented during the 2018 ESMO Congress, and the data revealed a trend toward improved response rates.

In the phase III IMvigor 130 trial, ${ }^{8}$ patients with locally advanced/unresectable or metastatic disease were randomly assigned in a 1:1:1 ratio to receive atezolizumab + platinum-based chemotherapy ( $\operatorname{arm} \mathrm{A}$ ), atezolizumab monotherapy (arm B), or placebo + platinum-based chemotherapy (arm C). In the intention-to-treat population, median duration of progression-free survival appeared to be longer in arm A than in arm C (8.2 vs 6.3 months; $P=.007$ ). However, this has not yet translated into an OS benefit. The phase Ib EV-103 trial of enfortumab vedotin + pembrolizumab demonstrated a high objective response rate: there was a $70 \%$ reduction in the tumor size. ${ }^{9}$ However, according to Dr. Siefker-Radtke, these results were based on a small sample size.

"Combination strategies combining chemotherapy with an ICI are not yet FDA-approved or incorporated in the NCCN Clinical Practice Guidelines in Oncology [NCCN Guidelines]," she noted.

\section{Maintenance Strategy With Avelumab}

ICIs have been used to prolong the benefits of first-line systemic chemotherapy. Interim analysis results from the phase III JAVELIN Bladder 100 trial showed that patients who achieved stable disease or better after frontline platinum-based chemotherapy seemed to derive an OS benefit from maintenance therapy with avelumab. ${ }^{10}$

The use of avelumab as a maintenance strategy is now FDA-approved. Per the NCCN Guidelines for Bladder Cancer, patients who achieve stable disease or better after receiving cisplatin or carboplatin may be administered maintenance therapy with avelumab until disease progression (Figure 1) ${ }^{11}$ Dr. Siefker-Radtke noted that several other ICIs have been evaluated in this setting; however, they are not yet FDA-approved.

\section{Sequencing Strategies}

Patients with urothelial tumors may benefit from frontline chemotherapy followed by an ICI at disease progression. In the KEYNOTE-045 trial, ${ }^{2}$ the objective response 


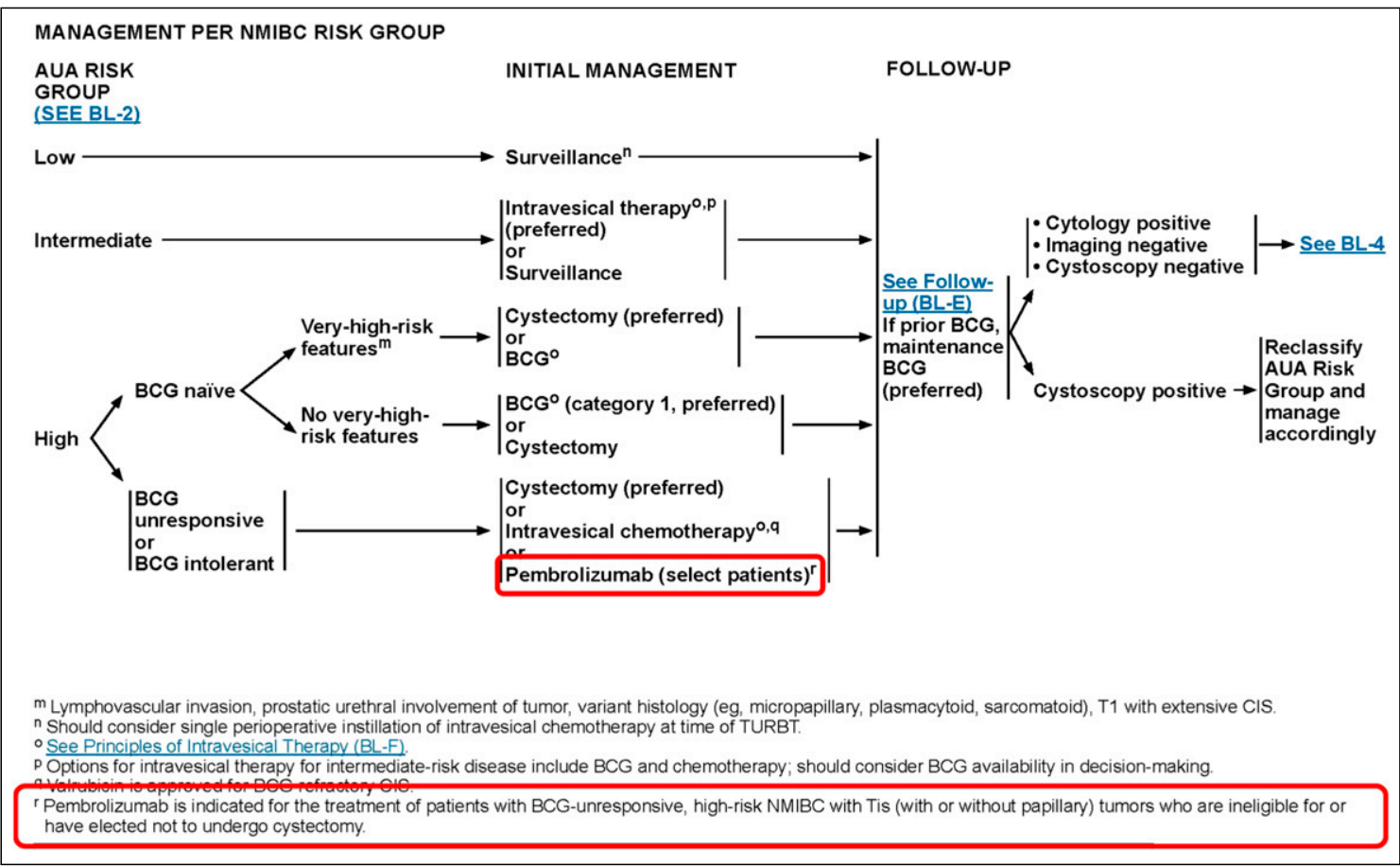

Figure 2. Management per risk group (BL-3). From the NCCN Guidelines for Bladder Cancer. Version 1.2021. To view the most recent version, visit NCCN.org.

(C) 2021 National Comprehensive Cancer Network (NCCN), All rights reserved. This illustration may not be reproduced in any form without the express written permission of NCCN.

rate appeared to be significantly higher with pembrolizumab than with taxane-based chemotherapy in patients with platinum-refractory advanced urothelial carcinoma (21.1\% vs $11.4 \% ; P=.001$ ). Median OS was 10.3 and 7.4 months, respectively (hazard ratio, $0.73 ; P=.002$ ). Pembrolizumab also exhibited an improved toxicity profile compared with taxane-based chemotherapy.

Based on the data from the KEYNOTE-045 study, the FDA approved the use of pembrolizumab in this clinical context. Shortly thereafter, it was integrated into the NCCN Guidelines as a preferred regimen. The ICIs nivolumab and avelumab have received early FDA approval also and are included in the NCCN Guidelines, ${ }^{11}$ although full approval may be granted after randomized trial data are obtained.

According to Dr. Siefker-Radtke, the multicenter phase III DANUBE study of durvalumab had a negative outcome without evidence of survival benefit. ${ }^{12}$ As a result, in February 2021 durvalumab was voluntarily withdrawn from the NCCN Guidelines for the secondline treatment of urothelial cancer.

\section{Immunotherapy for Adjuvant Urothelial Carcinoma}

CheckMate 274 is a double-blind, multicenter, phase III study, enrolling patients with high-risk muscle-invasive disease who have undergone radical surgery. Study participants were randomly assigned in a 1:1 ratio to receive nivolumab or placebo. There seemed to be early evidence of improvement in disease-free survival with nivolumab in the intention-to-treat and PD-L1-high populations $\left(P<.001\right.$ for both). ${ }^{13}$ The study remains immature for OS analysis. Additionally, according to Dr. Siefker-Radtke, the impact of this strategy on PD-L1-low tumors is not fully understood. As a result, adjuvant ICI is not FDA-approved and is not currently included in the NCCN Guidelines.

\section{Immunotherapy for Superficial Urothelial Cancer}

Based on the results of the KEYNOTE-057 trial, ${ }^{14}$ pembrolizumab was granted approval by the FDA and incorporated into the NCCN Guidelines for the treatment of patients with Bacillus Calmette-Guérin (BCG)-unresponsive, high-risk non-muscle-invasive bladder cancer (carcinoma in situ) who are ineligible for or have elected not to undergo cystectomy (Figure 2). Results of this phase II trial showed a 3-month complete response rate of $40.6 \%$, with a median duration of complete response of 16.2 months.

"We see approvals for maintenance strategies in response to chemotherapy, sequencing after [disease] progression from frontline chemotherapy, and also for the treatment of superficial urothelial cancer," Dr. Siefker-Radtke concluded. "There are multiple other settings where ICIs are currently under study and are 
showing promise. We hope to be able to add to the FDA approval list with the use of ICIs."

Disclosures: Dr. Siefker-Radtke has disclosed serving as a scientific advisor for AstraZeneca Pharmaceuticals LP, Bavarian Nordic, Bristol Myers Squibb
Company, Genentech, Inc., Immunomedics, Inc., Janssen Pharmaceutica Products, LP, Merck \& Co., Inc., Mirati Therapeutics, Inc., Nektar Therapeutics, Seattle Genetics, Inc., and Taiho Pharmaceuticals Co., Ltd

Correspondence: Arlene O. Siefker-Radtke, MD, The University of Texas MD Anderson Cancer Center, 1155 Hermann Pressler, Unit 1374, Houston, TX 77030. Email: asiefker@mdanderson.org

\section{References}

1. Siefker-Radtke AO, Millikan RE, Tu S, et al. Phase III trial of fluorouracil interferon alfa-2b, and cisplatin versus methotrexate, vinblastine, doxorubicin and cisplatin in metastatic or unresectable urothelial cancer. J Clin Oncol 2002;20:1361-1367.

2. Bellmunt J, de Wit R, Vaughn DJ, et al. Pembrolizumab as second-line therapy for advanced urothelial carcinoma. N Engl J Med 2017;376: 1015-1026.

3. Jiang $Q, F u ~ Q, C h a n g ~ Y$, et al. CD19+ tumor-infiltrating B-cells prime CD4+ T-cell immunity and predict platinum-based chemotherapy efficacy in muscle-invasive bladder cancer. Cancer Immunol Immunother 2019;68: 45-56.

4. Shin J, Chung J, Kim SH, et al. Effect of platinum-based chemotherapy on PD-L1 expression on tumor cells in non-small cell lung cancer. Cancer Res Treat 2019:51:1086-1097.

5. Galsky MD, Wang H, Hahn NM, et al. Phase 2 trial of gemcitabine, cisplatin, plus ipilimumab in patients with metastatic urothelial cancer and impact of DNA damage response gene mutations on outcomes. Eur Urol 2018;73:751-759.

6. Kim WS, Kim H, Kwon KW, et al. Cisplatin induces tolerogenic dendritic cells in response to TRL agonists via the abundant production of IL-10, thereby promoting $\mathrm{TH} 2$ - and Tr1-biased T-cell immunity. Oncotarget 2016;7:33765-33782.

7. Hoimes CJ, Albany C, Hoffman-Censits J, et al. Neoadjuvant pembrolizumab plus cisplatin and gemcitabine shows promise in locally advanced urothelial cancer [abstract]. Presented at the ESMO Congress 2018; October 19-23, 2018; Munich, Germany. Abstract LBA 33.

8. Grande E, Galsky MD, Arranz Arija JA, et al. IMvigor130: a phase III study of atezolizumab with or without platinum-based chemotherapy in previously untreated metastatic urothelial carcinoma [abstract]. Presented at the ESMO
Congress 2019; September 27-October 1, 2019; Barcelona, Spain. Abstract LBA 14.

9. Hoimes CJ, Rosenberg JE, Srinivas S, et al. EV-103: initial results of enfortumab vedotin plus pembrolizumab for locally advanced or metastatic urothelial carcinoma [abstract]. Presented at the ESMO Congress 2019; September 27-October 1, 2019; Barcelona, Spain. Abstract 4844.

10. Powles T, Park SH, Voog E, et al. Maintenance avelumab + best supportive care (BSC) versus BSC alone after platinum-based first-line (1L) chemotherapy in advanced urothelial carcinoma (UC): JAVELIN Bladder 100 phase III interim analysis [abstract]. Presented at the ASCO2O Virtual Scientific Program; May 29-31, 2020. Abstract LBA1.

11. Flaig TW, Spiess PE, Agarwal N, et al. NCCN Clinical Practice Guidelines in Oncology: Bladder Cancer. Version 2.2021. Accessed March 27, 2021. Available at NCCN.org

12. Powles $T$, van der Heijden MS, Castellano D, et al. Durvalumab alone and durvalumab plus tremelimumab versus chemotherapy in previously untreated patients with unresectable, locally advanced or metastatic urothelial carcinoma (DANUBE): a randomised, open-label, multicentre, phase 3 trial. Lancet 2020;21:1574-1588.

13. Bajorin DF, Witjes JA, Gschwend J, et al. First results from the phase 3 CheckMate 274 trial of adjuvant nivolumab vs placebo in patients who underwent radical surgery for high-risk muscle-invasive urothelial carcinoma [abstract]. Presented at the ASCO 2021 Genitourinary Cancers Symposium; February 11-13, 2021. Abstract 391.

14. Balar AV, Kamat AM, Kulkarni GS, et al. Pembrolizumab for the treatment of patients with Bacillus Calmette-Guérin unresponsive, high-risk non-muscle-invasive bladder cancer: over two years follow-up of KEYNOTE057 [abstract]. Presented at the ASCO20 Virtual Scientific Program; May 29-31, 2020. Abstract 5041. 